\title{
Fluid Flow-Induced Calcium Response in Osteoclasts: Signaling Pathways
}

\author{
Ping Li, ${ }^{1,2,3,4}$ Chenglin Liu, ${ }^{1}$ Man Hu, ${ }^{3}$ Mian Long, ${ }^{3}$ Ding Zhang, ${ }^{2}$ and Bo Huo ${ }^{1,3}$ \\ ${ }^{1}$ Department of Mechanics, School of Aerospace Engineering, Beijing Institute of Technology, No. 5 South Zhongguancun \\ Street, Beijing 100081, People's Republic of China; ${ }^{2}$ Department of Stomatology, Faculty of Surgery, Peking Union Medical \\ College Hospital, Peking Union Medical College, Chinese Academy of Medical Sciences, Beijing 100730, People's Republic of \\ China; ${ }^{3}$ Key Laboratory of Microgravity and Center for Biomechanics and Bioengineering, Institute of Mechanics, Chinese \\ Academy of Sciences, Beijing 100190, People's Republic of China; and ${ }^{4}$ Department of Maxillofacial \& E.N.T. Oncology, \\ Tianjin Medical University Cancer Institute and Hospital, Tianjin 300060, People's Republic of China
}

(Received 26 August 2013; accepted 25 January 2014; published online 8 April 2014)

Associate Editor Scott I. Simon oversaw the review of this article.

\begin{abstract}
Intracellular calcium oscillation and its downstream signaling in osteoclasts is believed to play critical roles in regulating bone resorption. Our previous study demonstrated that fluid shear stress (FSS) induced more calcium responsive peaks in the late differentiated osteoclasts than the early ones. In this paper, the signaling pathways of FSS-induced calcium response for the osteoclasts in different differentiation stages were studied. RAW264.7 macrophage cells were induced to differentiate into osteoclasts with the conditioned medium from MC3T3E1 osteoblasts. Furthermore pharmacological agents were added to block the specific signaling pathways. Finally the cells were exposed to FSS at different levels ( 1 or 10 dyne $/ \mathrm{cm}^{2}$ ) after being induced for 4 or 8 days. The results showed that the mechanosensitive, cation-selective channels, phospholipase C (PLC) and endoplasmic reticulum constituted the major signaling pathway for mechanical stimulation-induced calcium response in osteoclasts. Extracellular calcium or ATP involved with calcium oscillation in a FSS magnitude-dependent manner. This pathway study may help to give insight into the molecular mechanism of mechanical stimulation-regulated bone remodeling.
\end{abstract}

Keywords-Osteoclasts, Fluid shear stress, Calcium response, Signaling pathway, Differentiation.

\section{INTRODUCTION}

Bone marrow hematopoietic stem cells first differentiate into monocytes and then fuse into multinuclear

Address correspondence to Ding Zhang, Department of Stomatology, Faculty of Surgery, Peking Union Medical College Hospital, Peking Union Medical College, Chinese Academy of Medical Sciences, Beijing 100730, People's Republic of China and Bo Huo, Department of Mechanics, School of Aerospace Engineering, Beijing Institute of Technology, No. 5 South Zhongguancun Street, Beijing 100081, People's Republic of China. Electronic mails: dingz77@sina.com and huobo@bit.edu.cn osteoclasts which are responsible for resorbing bone minerals. During this differentiation process, the receptor activator of nuclear factor kappa-B ligand (RANKL) is demonstrated to induce the repetitive increase of cytosolic calcium ions concentration $\left[\mathrm{Ca}^{2+}\right]_{i}$, i.e., calcium oscillation, ${ }^{20}$ which is closely related to the differentiation of osteoclasts and their function in bone resorption. ${ }^{36}$ Osteoclasts are located on the bone surface and exposed to fluid filled within trabecular cavities. When mechanical loading is applied on bone, the deformation of bone matrix produces fluid shear stress (FSS) around osteoclasts and the in vivo studies concluded that FSS stimulates bone resorption mediated by osteoclasts. ${ }^{8,17}$ Our previous study demonstrated that FSS could induce potent calcium response and oscillation in osteoclasts and the properties of calcium response depended on the differentiation stages. ${ }^{23}$ Stronger calcium oscillation was found in small osteoclasts than the larger ones, and the calcium responsive sensitivity of osteoclasts to FSS was reduced along with the fusion of osteoclasts. But the signaling pathways of FSS-induced calcium response in osteoclasts remains unknown.

There are two sources for the increase of $\left[\mathrm{Ca}^{2+}\right]_{\mathrm{i}}$, i.e., the influx of extracellular calcium ions $\left(\left[\mathrm{Ca}^{2+}\right]_{0}\right)$ and the release of intracellular calcium stores. The extracellular calcium ions enter into a cell mainly through plasma membrane ion channels including mechanosensitive cation-selective channels (MSCC) or voltage sensitive calcium channels (VSCC) in osteoclasts. The preferred candidate of MSCC channels is transient receptor potential cation channel V4 (TRPV4), which mediated calcium influx to regulate the terminal differentiation of osteoclasts ${ }^{27}$ and bone remodeling. ${ }^{31}$ L-type VSCC channels express in osteoclasts and the calcium influx through them plays 
critical roles in RANKL-induced osteoclast formation by down-regulating nuclear factor of activated $\mathrm{T}$ cells c1 (NFATc1), a $\mathrm{Ca}^{2+}$-responsive transcription factor that drives osteoclastogenesis. ${ }^{33}$ One study showed that MSCC blocker gadolinium $\left(\mathrm{Gd}^{3+}\right)$ could inhibit actin ring formation in osteoclasts, whereas modulators of voltage-dependent $\mathrm{Ca}^{2+}$ channels had no effect. ${ }^{5}$ Another source of $\left[\mathrm{Ca}^{2+}\right]_{i}$ in osteoclasts is internal stores such as endoplasmic reticulum (ER). The extracellular stimuli like calcium ion or ATP activate the plasma membrane receptors, which further induces the phospholipase C (PLC) synthesis. ${ }^{19,29}$ PLC can activate inositol 1,4,5-trisphosphate (IP3) and IP3 triggers calcium release from ER via binding with IP3 receptors at ER membrane. ${ }^{7}$ It has been generally recognized that the repetitive release of $\mathrm{Ca}^{2+}$ from ER is the major reason of calcium oscillation that results in selective and robust induction of NFATc1. ${ }^{7,15,19}$

Lots of studies indicated that ATP could release from the mechanically stimulated osteoblasts or osteocytes and further binds the purinergic $\mathrm{P} 2$ receptor on the neighboring cells to cause the calcium release from ER. ${ }^{10,40}$ It has been found that the $\mathrm{P} 2 \mathrm{X} 7$ receptor is expressed in osteoclasts, ${ }^{21}$ which may contribute to cell-cell calcium transfer. The influence of $\mathrm{P} 2$ receptors on osteoclast physiology and bone physiology in general is beginning to be understood. ${ }^{35}$ But whether extracellular ATP could induce the $\left[\mathrm{Ca}^{2+}\right]_{\mathrm{i}}$ increase in a paracrine manner is still unknown although it was found that $\mathrm{P} 2 \mathrm{X} 7$ participated in osteoclast fusion. ${ }^{1,9,11}$ Gap junctional intercellular communication mediated by connexins is suggested to be another pathway of intercellular calcium transferring as the molecules smaller than $1.2 \mathrm{kDa}$ including IP3 or calcium ions can propagate within the cytosols among the neighboring cells. Connexin $43(\mathrm{Cx} 43)$, the gap junction proteins, has been found to be expressed in precursor or fully differentiated osteoclasts. ${ }^{37}$ Osteoclast formation and resorptive ability are also reported to be modulated by gap junction. ${ }^{2,16,28}$ But it still remains unknown whether this connexin-dependent behaviors of osteoclasts are involved with calcium response.

To clarify the signaling pathway of calcium response in osteoclasts under mechanical stimulation will be important for the understand of mechanotransduction in bone remodeling. In the present study, the differentiation of RAW264.7 macrophage cells to osteoclasts was induced and the calcium response of osteoclasts with different nuclear numbers $(1,2-5$ and $\geq 6$ ) at 4- or 8-days induction under different levels of FSS ( 1 and 10 dyne $/ \mathrm{cm}^{2}$ ) was observed. Meanwhile the abovementioned calcium signaling pathways, i.e., MSCC, VSCC, $\left[\mathrm{Ca}^{2+}\right]_{0}$, PLC, ER, ATP and gap junction, were studied.

\section{MATERIALS AND METHODS}

\section{Materials}

The RAW264.7 cells were purchased from the European Collection of Cell Cultures (ECACC, Wiltshire, UK). The MC3T3-E1 cells were purchased from the American Type Culture Collection (ATCC, Manassas, USA). Dulbecco's Modified Eagle's Medium (DMEM/ high glucose), alpha modified Minimum Essential Medium ( $\alpha$-MEM), phosphate buffered saline (PBS), trypsinEDTA, penicillin/streptomycin, and L-glutamine were provided by Hyclone (Thermo Scientific, Beijing, China). Dimethyl sulfoxide (DMSO), thapsigargin (TG), nifedipine, gadolinium chloride, $18 \beta$-glycyrrhetinic acid (GA) were obtained from Sigma (St. Louis, USA). Thermanox plastic slides were purchased from NUNC (Roskilde, Denmark). Fetal bovine serum (FBS), Fluo-4 AM, Pluronic F-127, and Hoechst 33342 were purchased from Invitrogen (Eugene, USA). Suramin and U73122 were purchased from Calbiochem (Darmstadt, Germany). DMEM High Glucose $\mathrm{Ca}^{2+}$-free medium were from Gibco (Carlsbad, USA).

\section{Induction of Osteoclast Differentiation by Conditioned Medium from Osteoblasts}

In the present study, we adopted the conditioned medium from MC3T3-E1 osteoblasts to induce the fusion of RAW264.7 monocytes, through which TRAPpositive, multinucleate osteoclasts have been demonstrated to form in our previous study. ${ }^{23}$ The osteoblasts were seeded in dishes at a density of $2 \times 10^{4} \mathrm{cells} / \mathrm{cm}^{2}$ and maintained in $\alpha$-MEM supplemented with $10 \%$ heatinactivated $\mathrm{FBS}$ and $1 \%$ penicillin-streptomycin solution at $37{ }^{\circ} \mathrm{C}$ in a humidified atmosphere of $5 \% \mathrm{CO}_{2}$. The medium was changed every $48 \mathrm{~h}$, and the conditioned medium was collected. RAW264.7 cells were seeded at a density of $2 \times 10^{4}$ cells $/ \mathrm{cm}^{2}$ on plastic slides. After $24 \mathrm{~h}$, the medium was replaced with a 1:1 (v/v) mixture of the conditioned medium and the growth medium of DMEM supplemented with $10 \%$ heat-inactivated FBS, $3.5 \mathrm{mM}$ L-glutamine, and $1 \%$ penicillin-streptomycin solution. The induction medium containing the conditioned medium was changed every other day. After 2 days induction, multinucleated osteoclasts will be observed and the nuclear number of one cell can be estimated by its spreading area as described in our previous paper ${ }^{23}$ (Fig. 1a).

\section{Intracellular Calcium Response Under FSS}

When RAW264.7 cells had been induced in the conditioned medium for 4- or 8-days, respectively, the cytosolic calcium ions in cell were stained with $5 \mu \mathrm{M}$ Fluo-4 AM in DMSO and $0.02 \%$ Pluronic F- 127 for $2 \mathrm{~h}$ 
(a)

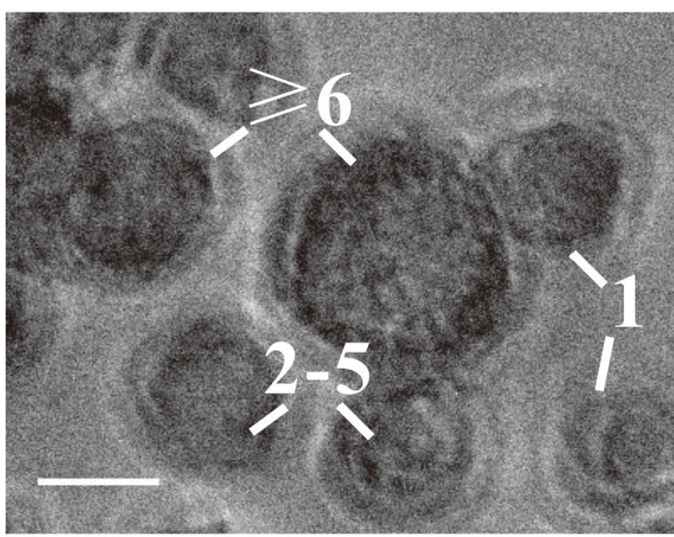

(c)

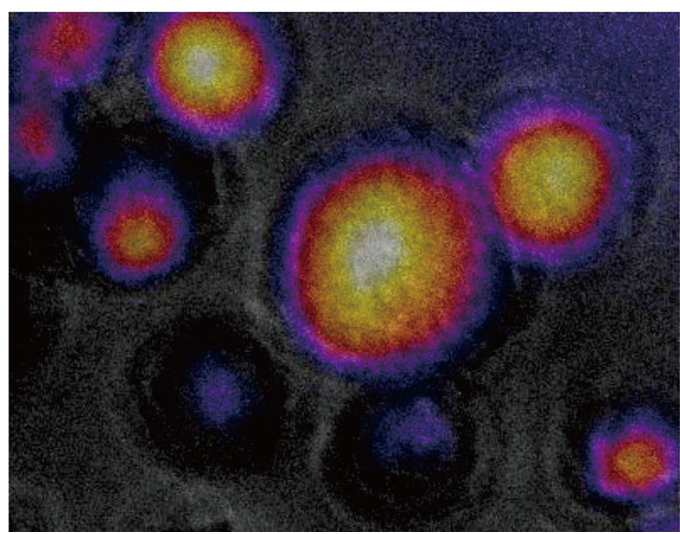

(b)

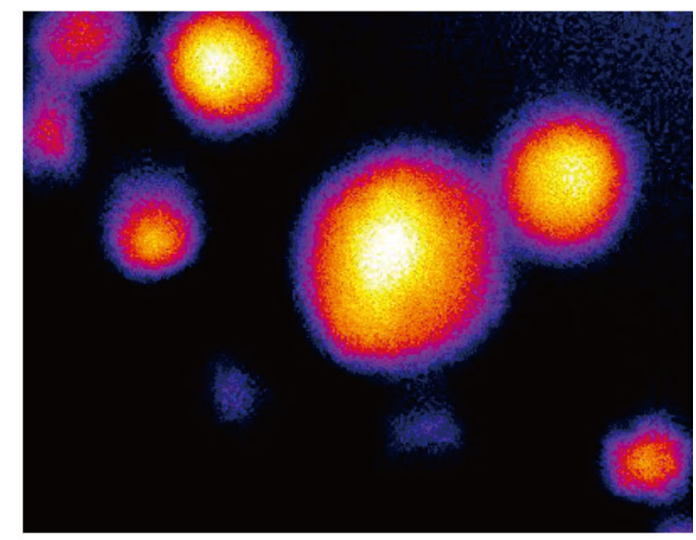

(d)

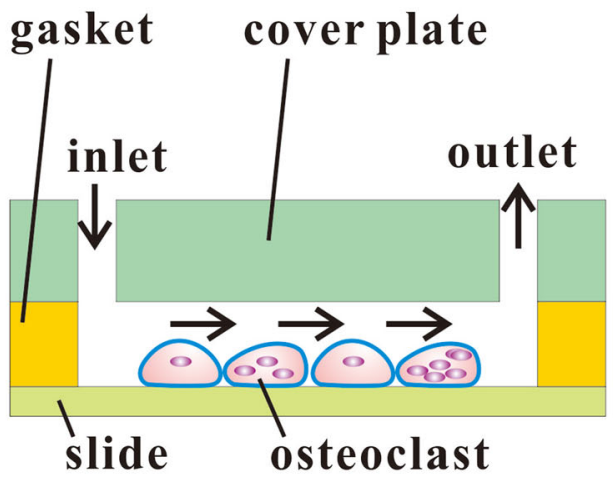

FIGURE 1. FSS exertion and calcium response in osteoclasts. (a) Bright field image of 8-days-induced osteoclasts, in which the digits indicate the number of nuclei estimated by cell area. ${ }^{23}$ (b) Heat map of Fluo-4 AM stained osteoclasts under FSS of 10 dyne/ $\mathrm{cm}^{2}$. (c) Merged image of (a) and (b). (d) Sketch map of the parallel plate flow chamber adopted in the present study.

in a culture medium at room temperature (Fig. 1b). The slide with cells was then rinsed with dye-free medium twice, and was mounted in a custom-made parallel plate flow chamber (Fig. 1d). The entire operation was performed with extreme caution to minimize the response of cells to early agitations. When flow chamber is placed under microscope, 20-min resting period was sustained to make cells recover the stable state for repetitive calcium responses. The calcium response of cells under the steady fluid flow stimulation was recorded with CCD for $10 \mathrm{~min}$ including $1 \mathrm{~min}$ for baseline followed by the 9-min FSS stimulation. The fluorescence intensity of the calcium concentration for each cell was later normalized by its corresponding baseline (Fig. 2).

\section{INHIBITION OF CALCIUM RESPONSE PATHWAYS}

Besides the untreated group, seven different pharmacological agents were employed to treat the cells before and during experiments in order to identify the signaling pathways of intercellular calcium response in osteoclasts. For each test, osteoclasts on the surface of plastic slides were incubated in a specific inhibitor solution mixing with $5 \mu \mathrm{M}$ Fluo-4 AM dye medium for a certain period and the inhibitor would be contained in flow medium during FSS stimulation. $10 \mu \mathrm{M} \mathrm{Gd}^{3+}$ was supplied as the MSCC blocker and the incubation period was $10 \mathrm{~min}$. $5 \mu \mathrm{M}$ nifedipine was used to block L-type VSCC channels for $10 \mathrm{~min}$ incubation. Calcium-free DMEM medium replacing the regular medium was to remove the extracellular calcium. $10 \mu \mathrm{M} \mathrm{U} 73122$ was adopted to inhibit PLC for $10 \mathrm{~min}$ incubation. The calcium stored in ER was depleted by $1 \mu \mathrm{M}$ TG by $10 \mathrm{~min}$ incubation. $100 \mu \mathrm{M}$ suramin was employed to block the binding of ATP with P2 purinergic receptors by $30 \mathrm{~min}$ incubation. Intercellular gap junctional transferring was blocked by incubating $75 \mu \mathrm{M}$ GA for $10 \mathrm{~min}$. 
Flow

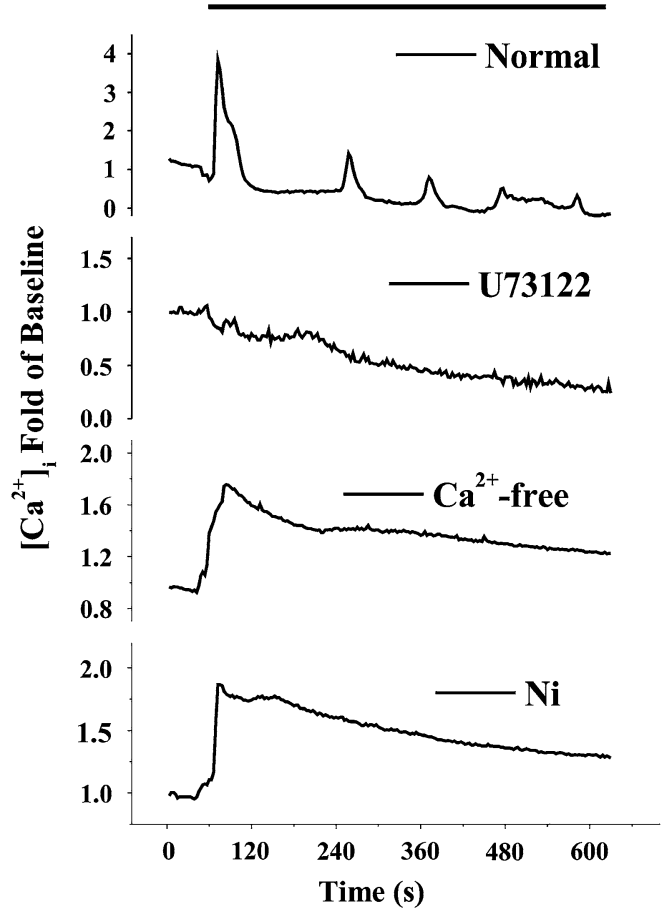

Flow

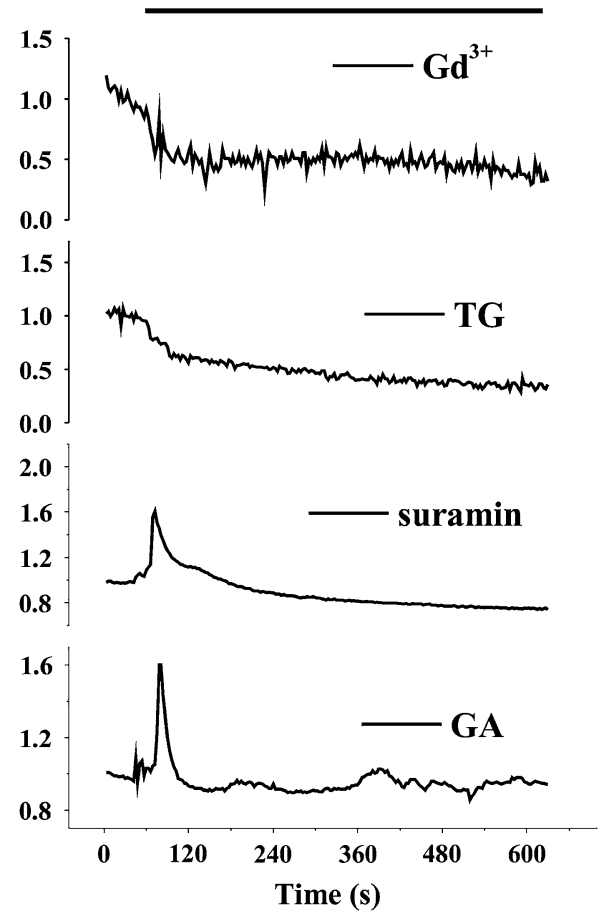

FIGURE 2. The typical curves of calcium response in osteoclasts under FSS. Different chemicals were added into culture medium before flow to block the specific calcium signaling pathways.

\section{Statistical Analysis}

More than 3 slides were tested for a specific group with different induction time, FSS level and inhibitor. In one group, 50-340 monocytes, 8-94 osteoclasts with $2-5$ nuclei, and $5-14$ osteoclasts with more than $\geq 6$ nuclei were analyzed. All the results were expressed as mean \pm standard error of the mean (SEM). Statistical differences between the mean values of different groups were determined using one-way ANOVA, and was considered significant when $P<0.05$.

\section{RESULTS}

\section{MSCC-PLC-ER is the Primary Pathway of FSS- Induced Calcium Response}

As it had been clarified in our previous study, most monocytes or 2-5 nuclei-cells in the untreated group could respond to FSS stimulation although the responsive percentage significantly decreased for the larger multinucleate osteoclasts with more than six nuclei. ${ }^{23}$ At the low level of $1 \mathrm{dyne} / \mathrm{cm}^{2}$, when inhibiting MSCC channels by using $\mathrm{Gd}^{3+}$, or blocking PLC by U73122, or depleting ER calcium store by TG, only 4-7\% cells after 4-days induction could respond in spite of their nuclear number (Fig. 3). In case removing extracellular calcium by using calcium-free medium, responsive percentage was about $45 \%$ for monocytes or medium-size osteoclasts (2-5 nuclei), but decreased to $36 \%$ for the larger osteoclasts with $\geq 6$ nuclei. However, no significant difference was detected for most cells with different nuclei when blocking calcium signaling pathways.

At high FSS level of 10 dyne $/ \mathrm{cm}^{2}$, MSCC-PLC-ER is still the primary axis of FSS-induced calcium response and blocking either one of three pathways makes only less than $10 \%$ cells capable of responding. It is surprisingly found, however, that enhancing FSS level significantly increased the responsive percentage in the absence of $\left[\mathrm{Ca}^{2+}\right]_{0}$ especially for monocytes or 2-5 nuclei osteoclasts if compared with low FSS level. The significant difference between FSS levels is also detected for ATP-blocked group for monocytes or the larger osteoclasts with $\geq 6$ nuclei and for gap junctionblocked group for the medium- or large-size osteoclasts. The results also showed that the VSCC-blocked group is FSS-insensitive.

FSS-Induced Calcium Oscillation depends on $\left[\mathrm{Ca}^{2+}\right]_{0}$-MSCC-PLC-ER Pathway and FSS Level

Figure 4 shows that after 4-days induction, the peak's number of calcium response for the responsive cells to FSS stimulation for $\mathrm{Ca}^{2+}$-free, ATP-, VSCCand gap junction-blocked groups. At low FSS level of 

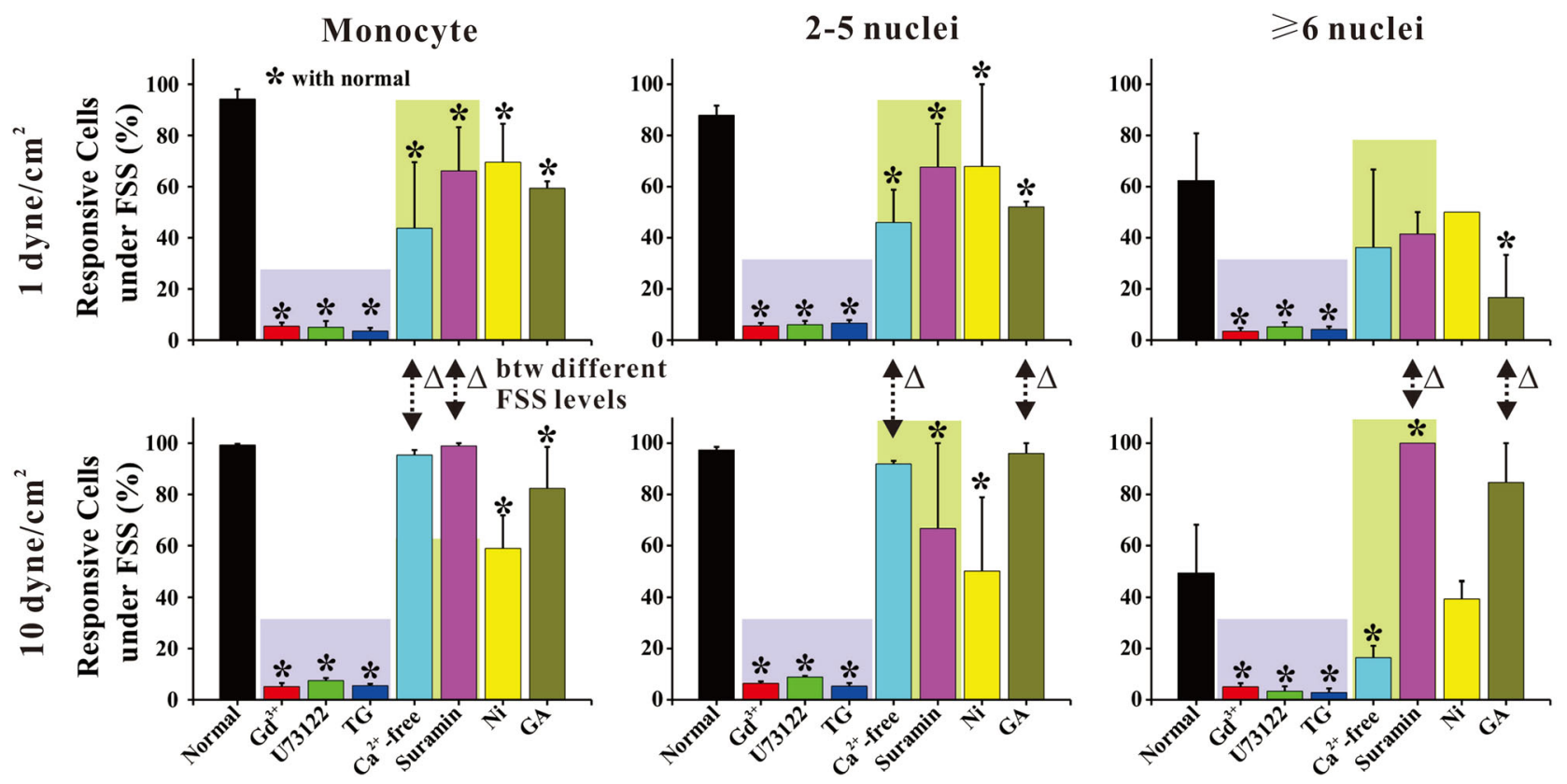

FIGURE 3. The non-responsive percentage of osteoclasts with different nuclei after 4-days induction under two levels of FSS when blocking the different calcium signaling pathways. The rectangular background blocks in pink-blue or moon green display the similar responses. The data are represented as mean \pm standard error. The double-head arrows display the significant difference between low and high FSS levels $(\Delta)\left({ }^{*}, \Delta, p<0.05\right)$.

1 dyne $/ \mathrm{cm}^{2}$, removing extracellular calcium led to only one responsive peak in 9-min FSS stimulation. Blocking other three pathways didn't significantly influence the calcium oscillation for monocytes or 2-5 nuclei osteoclasts compared with normal group, in which there were about $30 \%$ of cells responded more than once. All groups only had one responsive peak for the multinucleate osteoclasts with $\geq 6$ nuclei. When FSS level increased to $10 \mathrm{dyne} / \mathrm{cm}^{2}$, the normal group can respond twice averagely while there was only one peak in the absence of $\left[\mathrm{Ca}^{2+}\right]_{0}$ independent of nuclear number. Compared with the low FSS level, the significant difference occurred in ATP-blocked group for monocytes and in ATP- or VSCC-blocked groups for 2-5 nuclei osteoclasts, in which responsive peaks' number was reduced to one at high FSS level.

\section{Long-Term Induced Osteoclasts Depends on MSCC- PLC-ER Pathway to Produce Calcium Response}

When RAW264.7 precursors were induced to differentiate for 8 days, MSCC-PLC-ER is still the primary pathway for $\left[\mathrm{Ca}^{2+}\right]_{\mathrm{i}}$ response (Fig. 5a). In case of blocking either one of MSCC, PLC or ER, only $5 \%$ of cells could respond to FSS stimulations, independent of FSS level or nuclear number. If removing extracellular calcium, a large number of monocytes or $2-5$ nuclei cells could respond although most of large multinucleate osteoclasts with $\geq 6$ nuclei did not have response. Similar to 4-days induction, increasing FSS level from 1 to 10 dyne $/ \mathrm{cm}^{2}$ increased the responsive percentage from $41 \%$ to $89 \%$ for monocytes and from $67 \%$ to $81 \%$ for $2-5$ nuclei cells. In those responsive cells, almost all of cells only have one responsive peak in spite of FSS level and nuclear number when removing extracellular calcium (Fig. 5b). It indicates that calcium oscillation disappeared in the absence of $\left[\mathrm{Ca}^{2+}\right]_{0}$. But blocking ATP pathway seemed not to influence the ability of calcium oscillation for monocytes or 2-5 nuclei osteoclasts, especially under 10 dyne $/ \mathrm{cm}^{2}$ FSS level.

\section{The Temporal Characters of the First Responsive Peak} are Regulated by Induction Duration and FSS Level

Figure 6a showed the time to the first peak of cytosolic calcium response. At low FSS level and 4-days induction, the responding cells in normal group needed $49 \mathrm{~s}$ (monocytes), $71 \mathrm{~s}$ ( $2-5$ nuclei) and $86 \mathrm{~s}$ ( $\geq 6$ nuclei) to reach their first responsive peak after being exposed to FSS stimulation. The $\left[\mathrm{Ca}^{2+}\right]_{0}$-removed group spent much longer time of $177,111,278 \mathrm{~s}$ for osteoclasts with different nuclear number. Similarly, the average times to the first peak for ATP-blocked group are 203, 247 and 283 s, respectively. When RAW264.7 cells were induced to differentiate for a longer time of 8 days, the time to the first peak varied from $44 \mathrm{~s}$ to $134 \mathrm{~s}$, whose sensitivity to FSS stimulation isn't similar to 4-days induction after $\left[\mathrm{Ca}^{2+}\right]_{0}$ or ATP pathways were blocked. At high FSS level of 10 dyne $/ \mathrm{cm}^{2}$, the sensitivity of cells to 

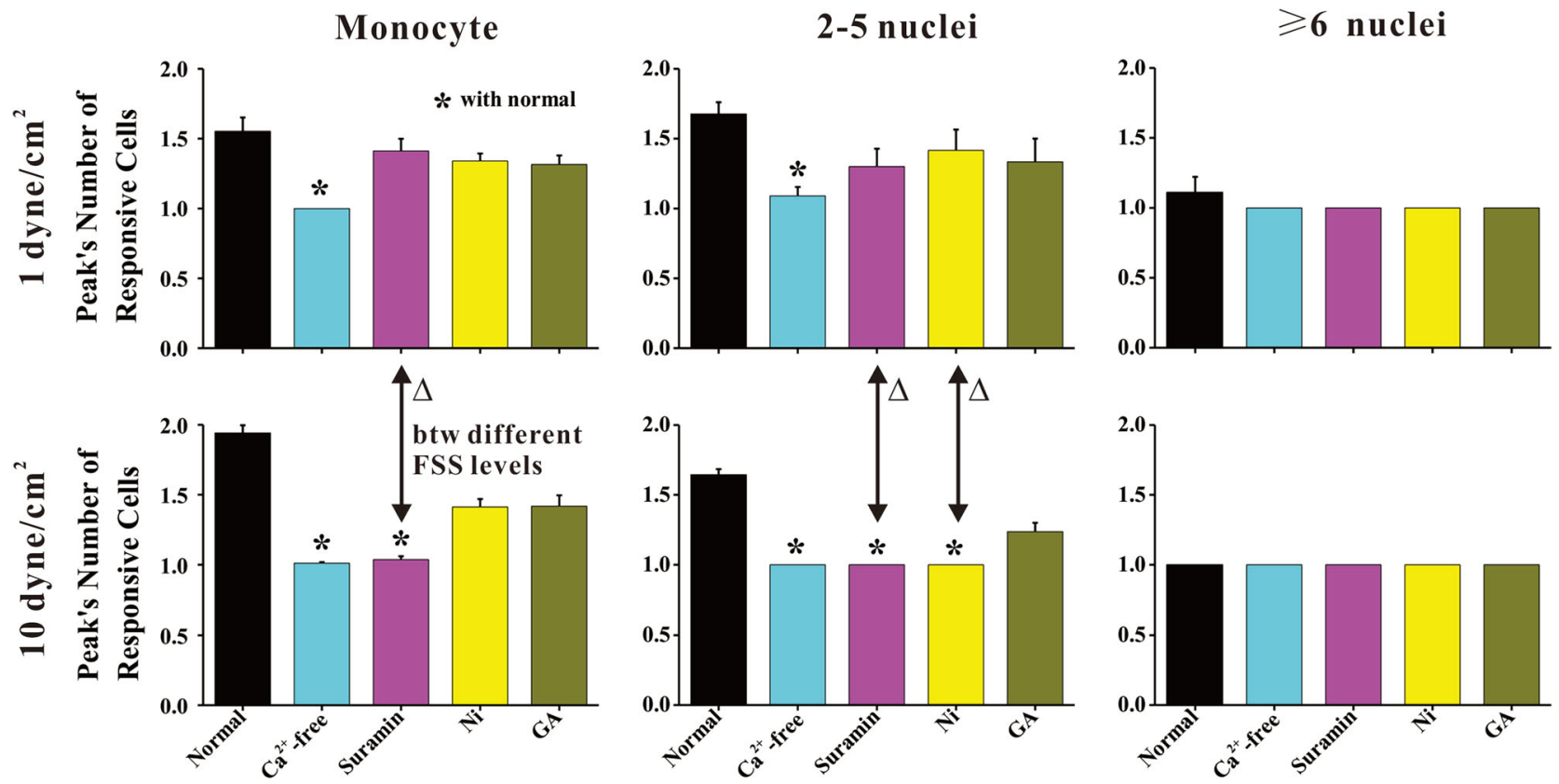

FIGURE 4. The peak's number of calcium response in those responsive osteoclasts with different nuclei at 4-days induction under two levels of FSS when blocking different calcium signaling pathways. The data are represented as mean \pm standard error. The double-head arrows display the significant difference between low and high FSS levels $(\Delta)\left({ }^{*}, \Delta, p<0.05\right)$.

FSS stimulation was significantly improved. Except for $58-81 \mathrm{~s}$ for the large osteoclasts ( $\geq 6$ nuclei) after 4-days induction, the time to the first peak varied from 15 to $36 \mathrm{~s}$ independent of induction duration and nuclear number.

After the cytosolic calcium concentration reached its peak value, the calcium ions will be uptaken back to ER or flux into the extracellular environment, which leads to the decrease of $\left[\mathrm{Ca}^{2+}\right]_{\mathrm{i}}$. The time interval between the first peak to its $50 \%$ value reflects the relaxation rate of the first peak. For the cells under same FSS stimulation and induction duration, the relaxation time of the first peak increased along with the nuclear number. But when extracellular calcium and ATP pathways were blocked, this tendency was not that significant. Increasing FSS level significantly shortened the relaxation time of normal group. In addition, at high FSS level, the significant difference between 4- and 8-days induction groups was detected but not at low FSS level for most cells.

\section{DISCUSSION}

The present study indicated MSCC-PLC-ER as the primary signaling pathway of $\left[\mathrm{Ca}^{2+} l_{i}\right.$ response in osteoclasts under FSS stimulations (Fig. 7). To our knowledge, there have been only a few studies on the mechanotransduction of calcium response in osteoclasts. For example, Xia and Ferrier made use of micropipette to apply single-cell indentation on isolated rabbit osteoclasts and they found that the mechanical perturbation-induced $\left[\mathrm{Ca}^{2+} 1_{i}\right.$ increase was inhibited by T-type VSCC inhibitor nickel chloride $\left(\mathrm{Ni}^{2+}\right)$ but not by MSCC inhibitor $\mathrm{Gd}^{3+}$ or L-type VSCC inhibitors nifedipine or verapamil. ${ }^{44}$ Comparing with this single-cell indentation study, our present data showed similar L-type VSCC-independent $\left[\mathrm{Ca}^{2+} 1_{\mathrm{i}}\right.$ response under FSS stimulation, but revealed a converse phenomenon for MSCC blocking as $\mathrm{Gd}^{3+}$ completely blocked the calcium response. Whether the type of mechanical stimulation causes different activation status of MSCC channels needs further clarification. In another micropipette indentation study, the hydrolysis of extracellular ATP by nucleotidase apyrase did not inhibit deformation-induced elevation of $\left[\mathrm{Ca}^{2+} 1_{i}\right.$ in the presence of extracellular $\mathrm{Ca}^{2+}$, indicating that nucleotide release is not essential for mechanically induced $\mathrm{Ca}^{2+}$ influx, ${ }^{42}$ which is similar to our present study. Except for the above two studies, there has not been any investigation concerning the signaling pathways of mechanical stimulation-induced calcium response in osteoclasts, although a few studies did observe the $\left[\mathrm{Ca}^{2+} 1_{\mathrm{i}}\right.$ response in osteoclasts induced by single-cell indentation through a glass pipette, ${ }^{45}$ whole-cell membrane stretching by changing the osmotic pressure stretching ${ }^{41}$ or FSS stimulation in our previous study. ${ }^{23}$

It has been recognized very early that when stretching osteoclasts, calcium-conducting ion chan- 
(a)

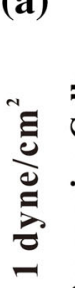

Monocyte

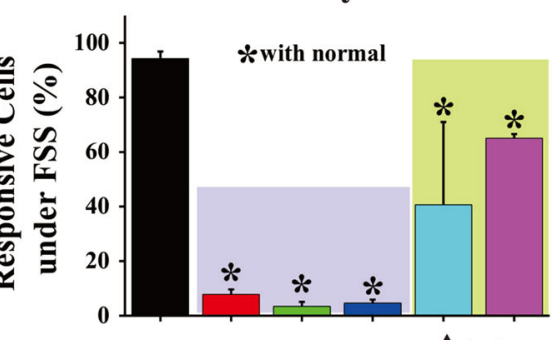

$\Delta$ btw different

FSS levels

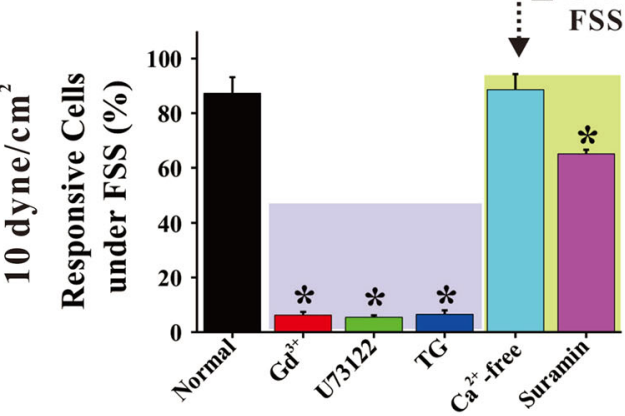

(b)
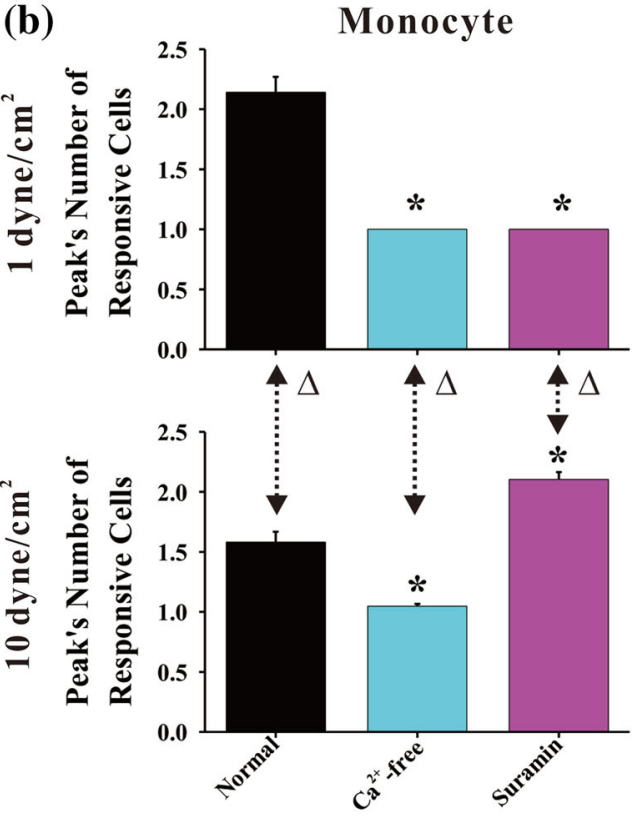

2-5 nuclei
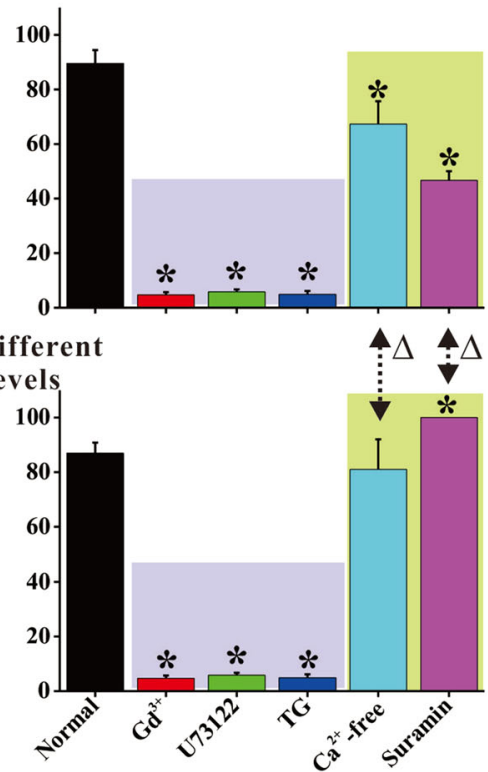

2-5 nuclei

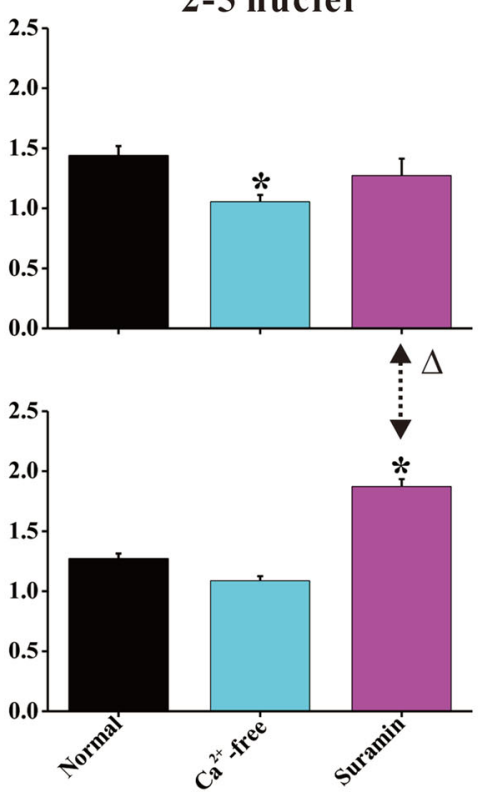

$\geqslant 6$ nuclei
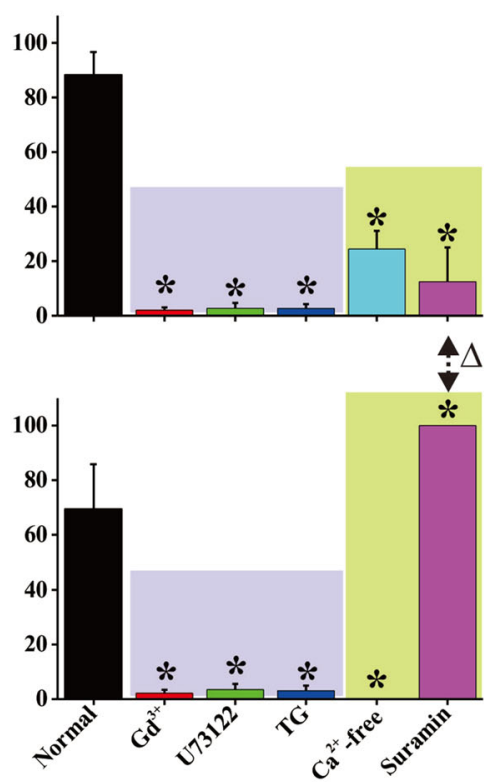

$\geqslant 6$ nuclei

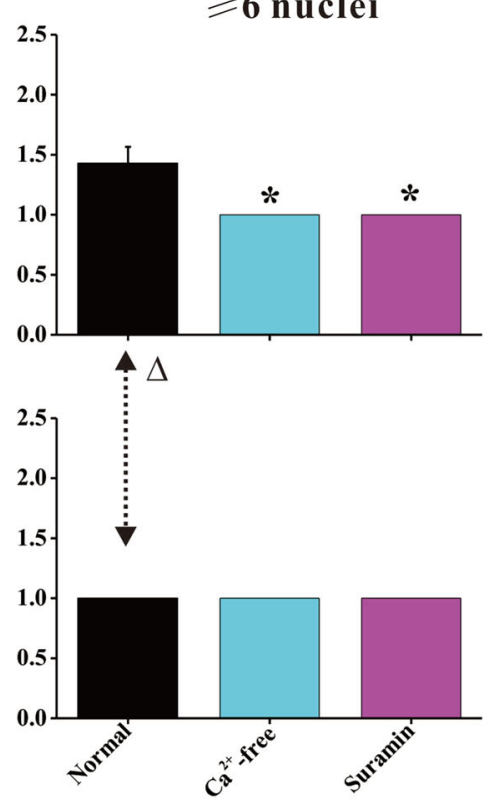

FIGURE 5. The non-responsive percentage (a) and peak's number (b) of osteoclasts with different nuclei for 8-days induction under two levels of FSS when blocking the different calcium signaling pathways. The rectangular background blocks in pink-blue or moon green display the similar responses. The double-head arrows display the significant difference between low and high FSS levels $(\Delta)$. The data are represented as mean \pm standard error $\left({ }^{*}, \Delta, p<0.05\right)$.

nels on cell membrane are activated. ${ }^{43}$ Transient receptor potential (TRP) channels are non-selective cation channels with significant $\mathrm{Ca}^{2+}$ permeability. ${ }^{39}$ One TRP subfamily member, TRPV4, is proposed to be the most possible candidate of MSCC channel on osteoclasts. ${ }^{6,34,39}$ In an in vivo study, TRPV4 deficiency suppressed unloading-induced osteoclastogenesis, i.e., reducing the levels of mineral apposition rate and bone formation rate, as well as increasing the number of osteoclasts. ${ }^{31}$ The further study focusing on the roles of TRPV4 in FSS-induced calcium response and migration of osteoclasts is in progress in our lab.

The present data showed that either depleting calcium ions in ER or blocking PLC was able to completely suppress the FSS-induced calcium response in osteoclasts. The signaling pathway of PLC activatedcalcium release from ER has been discovered in wide cell lineages such as endothelial cells, ${ }^{24}$ neurons, ${ }^{46}$ 
(a)
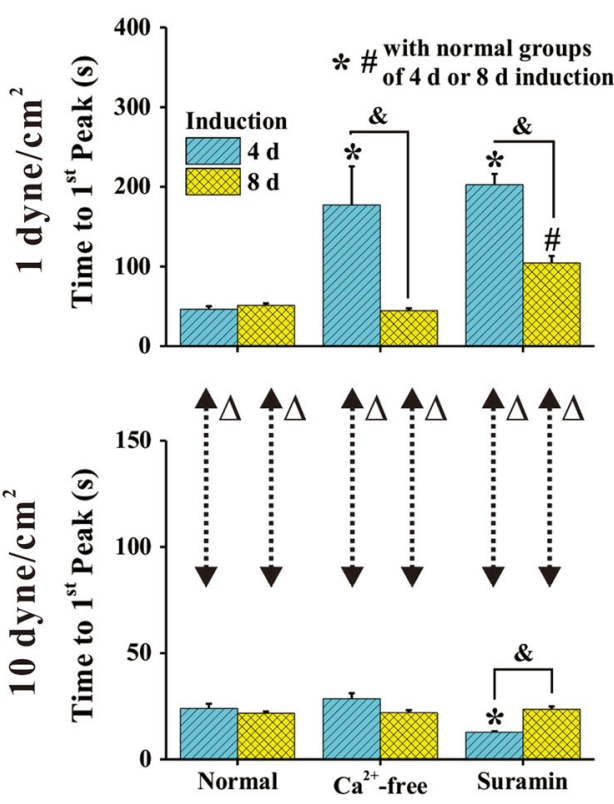

(b)
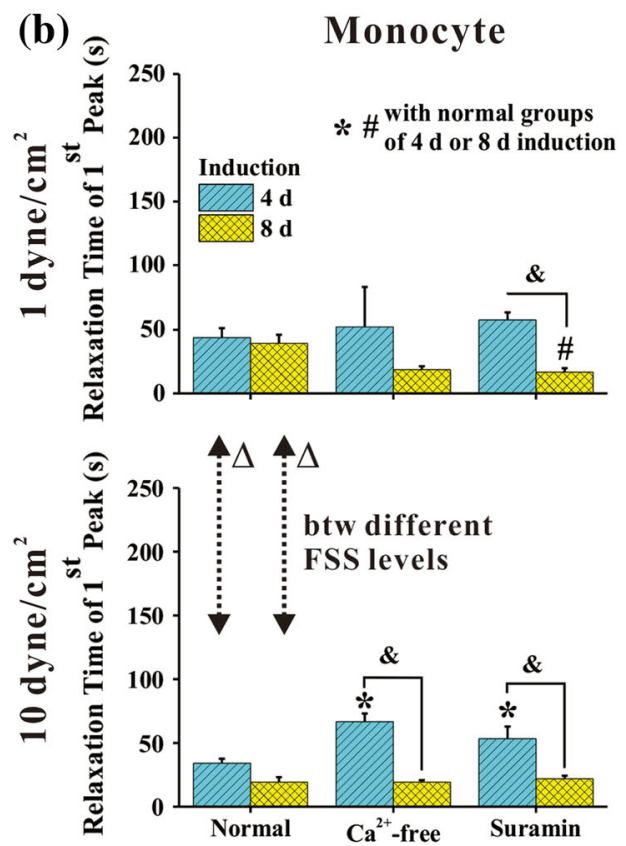

2-5 nuclei
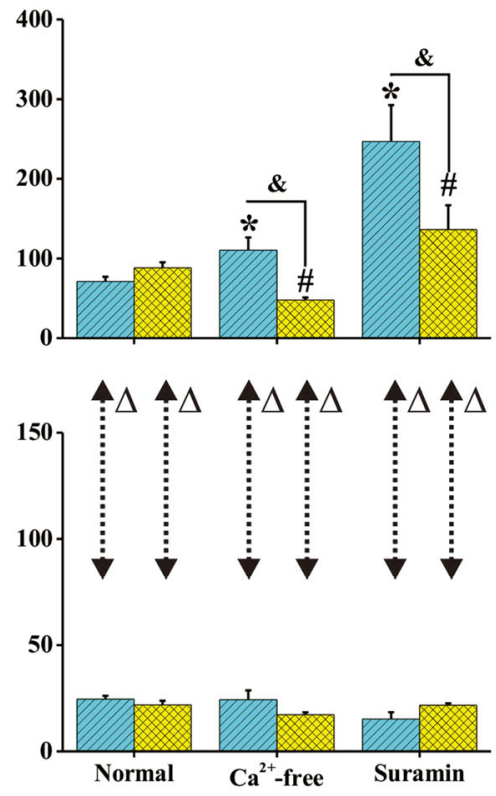

2-5 nuclei
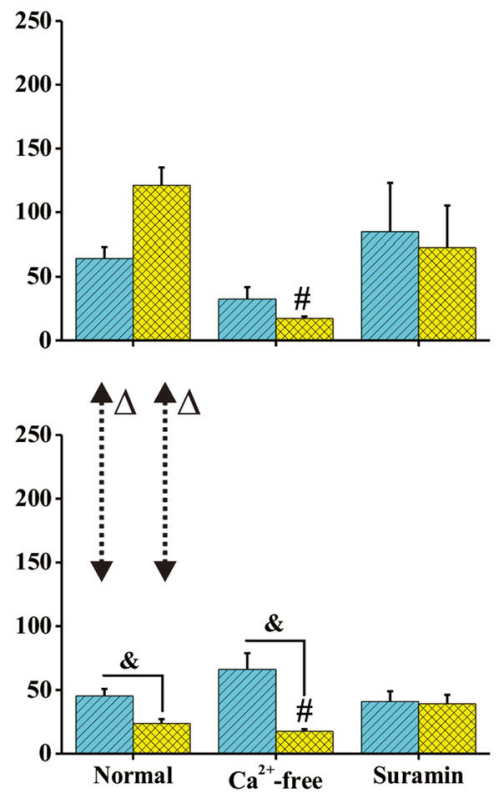

$\geqslant 6$ nuclei
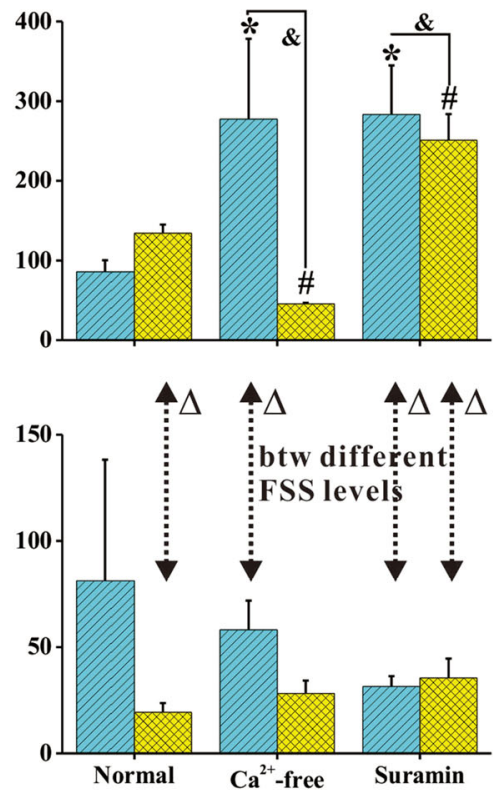

$\geqslant 6$ nuclei
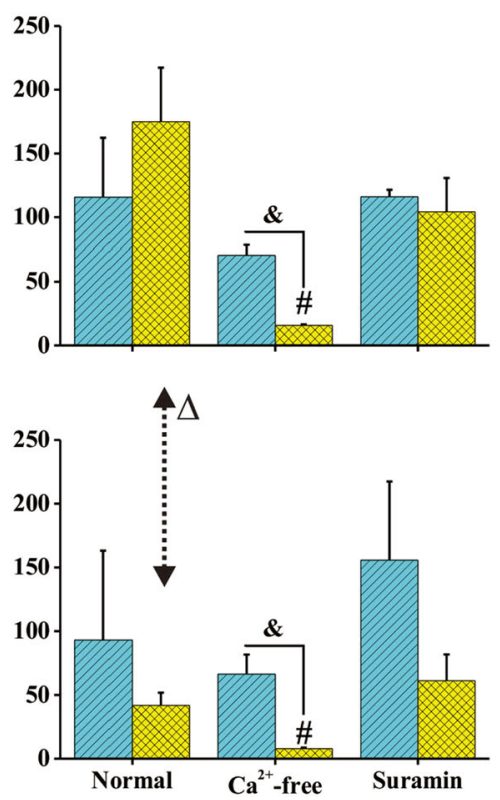

FIGURE 6. The time to the first response peak (a) and its $50 \%$ relaxation time (b) in those responsive osteoclasts with different nuclei at 4- and 8-days induction under two levels of FSS. The double-head arrows display the significant difference between low and high FSS levels $(\Delta)$. The data are represented as mean \pm standard error $\left(\&, \#,{ }^{*}, \Delta, \boldsymbol{p}<0.05\right)$.

osteoblasts $^{32}$ as well as osteoclasts. ${ }^{38}$ For this signaling pathway, PLC first activates IP3 and then IP3 binds with IP3R in ER membrane finally leading to the efflux of calcium from ER stores. Considering our results that blocking MSCC completely suppressed calcium response, we may infer that MSCC is coupled with PLC-ER signaling pathway. But the coupling relation of MSCC and PLC is still a little confusing. One study showed that the activation of PLC by the Gq proteins promoted the opening of the light activated channels TRPL. ${ }^{22}$ In addition, it was found that high extracellular calcium induced the increase of $\left[\mathrm{Ca}^{2+}\right]_{i}$, which could be blocked when inhibiting PLC even after depleting ER by thapsigargin. ${ }^{3}$ The above results suggest that calcium influx is PLC-dependent but store-insensitive and PLC might be linked to TRP 


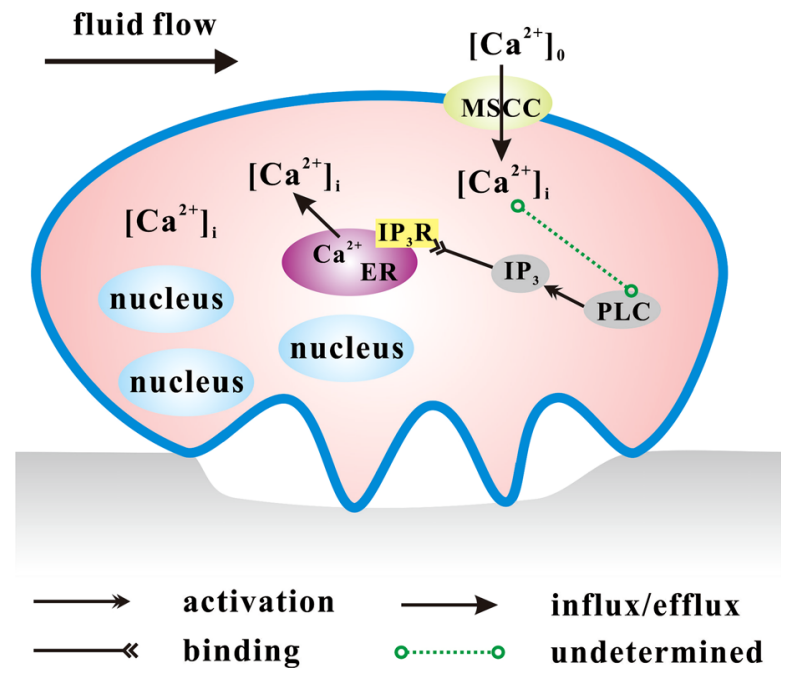

FIGURE 7. The schematic figure of FSS-induced calcium signaling pathways of osteoclasts.

channels. But our present results showed that FSSinduced $\left[\mathrm{Ca}^{2+}\right]_{\mathrm{i}}$ increase is obviously store-sensitive as blocking ER completely suppressed the $\left[\mathrm{Ca}^{2+}\right]_{\mathrm{i}}$ response. The further studies should be done to clarify which one is upstream, MSCC or PLC.

One notable result of this study is that when blocking ATP or gap junction pathways or removing extracellular calcium, the percentage of responsive osteoclasts increased along with the increase of FSS levels (Fig. 3). Considering the fact that MSCCblocking-suppressed $\left[\mathrm{Ca}^{2+}\right]_{\mathrm{i}}$ response is independent of FSS level, we hypothesized that this FSS dosedependent phenomenon must take effect through MSCC, i.e., that high FSS may directly open more MSCC channels. Although we used calcium-free medium to remove the extracellular calcium ions, there might be some intracellular calcium ions leaking into extracellular space, which may further go back into cells through ion channels to cause calcium response. Further investigations using EGTA to completely chelate the extracellular calcium ions should be carried out. In the absence of extracellular calcium, only one peak was found for those responsive osteoclasts implying that no calcium oscillation occurred (Fig. 4). Considering that depleting ER store with thapsigargin completely suppressed calcium response, we conclude that extracellular calcium influx triggers the calcium oscillation, for which intracellular stores are responsible (Fig. 4). This conclusion is coincided with the recent reports that RANKL-induced $\left[\mathrm{Ca}^{2+}\right]_{i}$ oscillation involved not only repetitive intracellular $\mathrm{Ca}^{2+}$ release from ER but also via store-operated $\mathrm{Ca}^{2+}$ entry TRPV channels during osteoclast differentiation. ${ }^{18} \mathrm{It}$ should be noted that the magnitude of the first peak of calcium response of osteoclasts induced by FSS is generally higher than the subsequent peaks, which is not similar to the persistent calcium oscillation stimulated by RANKL (Kuroda, Hisatsune et al. 2008). The molecular mechanism of this difference still needs further investigations.

It was demonstrated that osteoclasts constitutively released ATP via the $\mathrm{P} 2 \mathrm{X} 7$ receptor in response to a variety of stimuli including mechanical stimulation. ${ }^{4,35}$ The released ATP negatively regulated bone-resorbing activity through an autocrine/paracrine feedback loop. ${ }^{21,30}$ When the extracellular ATP was hydrolyzed by nucleotidase apyrase, the micropipette indentationinduced elevation of $\left[\mathrm{Ca}^{2+} 1_{i}\right.$ didn't change, indicating that nucleotide release is not essential for mechanically induced $\mathrm{Ca}^{2+}$ influx. ${ }^{42}$ We blocked the binding of ATP to the purinergic receptor with suramin and found that responsive percentage of osteoclasts decreased at low FSS level while almost all of cells responded for high FSS level (Fig. 3). Especially for the cells with $\geq 6$ nuclei under 10 dyne $/ \mathrm{cm}^{2}$ FSS (Fig. 3), the addition of suramin increased the number of responding cells compared with the normal group. One explanation for this unexpected phenomenon is that the cells with $\geq 6$ nuclei are much less than the smaller osteoclasts, which may lead to inconsistent results. Therefore it seems that high FSS stimulation-induced $\left[\mathrm{Ca}^{2+} 1_{i}\right.$ doesn't depend on ATP pathway, similar to the above indentation study. Our previous studies have demonstrated that ATP played a critical role on calcium oscillation for osteoblasts ${ }^{12-14}$ or osteocytes ${ }^{25,26}$ under mechanical stimulation such as fluid flow or AFM indentation. The present data showed that blocking ATP pathway abolished calcium oscillation for 4-days-induced osteoclasts under high FSS level (Fig. 4), however, 8-days induction group conversely revealed more obvious calcium oscillation compared with the normal group without inhibitors (Fig. 5). These opposite results may be relating to the fact that the amount of ATP release or the expression level of $\mathrm{P} 2 \mathrm{X} 7$ in mature cells is one-sixth or fourfold of that in precursors, respectively. ${ }^{4}$ The higher FSS might activate other signaling pathways that is responsible in calcium oscillation such as MSCC membrane channels or deformation-related intracellular pathways. However, how ATP pathway regulates FSS-induced calcium oscillation or intercellular calcium transfer needs more investigations.

When RAW264.7 precursor were induced to differentiation into multinucleate osteoclasts for 4- or 8 -days, the present study showed that along with the fusion, cells needed longer time to respond and restore (Fig. 6) and the detailed discussions can be referred to our previous study. ${ }^{23}$ But when blocking the calcium signaling pathways such as ATP or extracellular calcium, 4- and 8-days induction groups revealed a 
significantly different responding profile of the first peak (Fig. 6). It seems that 4-days-induced cells take longer time to respond to FSS stimulation but longer induction group doesn't. To clarify the signaling transduction mechanism of FSS-induced calcium response of osteoclasts in different differentiation stages will depend on the accumulation of experimental data.

In conclusion, the results of this study suggest that FSS stimulation activates MSCC channels and PLC-ER pathways, either of which is necessary for $\left[\mathrm{Ca}^{2+}\right]_{\mathrm{i}}$ response. Extracellular calcium, VSCC and ATP pathways involves with FSS-induced $\left[\mathrm{Ca}^{2+}\right]_{\mathrm{i}}$ response and oscillation in a FSS dose-dependent manner. Osteoclasts after different induction durations reveal significantly different temporal properties of responsive peak.

\section{ACKNOWLEDGMENT}

This work was supported by the National Natural Science Foundation of China [11372043 (BH) and 31070829 (DZ)], National Key Basic Research Foundation of China grant [2011CB710904 (ML)] and the Fundamental Research Funds for the Central Universities [GZ2013015101 (BH)].

\section{CONFLICT OF INTEREST}

No conflict of interested is assigned to the manuscript.

\section{REFERENCES}

\footnotetext{
${ }^{1}$ Adinolfi, E., F. Amoroso, and A. L. Giuliani. P2X7 receptor function in bone-related cancer. J. Osteoporos. 2012:637863, 2012.

${ }^{2}$ Batra, N., R. Kar, and J. X. Jiang. Gap junctions and hemichannels in signal transmission, function and development of bone. Biochim. Biophys. Acta 1818(8): 1909-1918, 2012.

${ }^{3}$ Bennett, B. D., U. Alvarez, and K. A. Hruska. Receptoroperated osteoclast calcium sensing. Endocrinology 142(5):1968-1974, 2001.

${ }^{4}$ Brandao-Burch, A., et al. The $\mathrm{P} 2 \mathrm{X} 7$ receptor is an important regulator of extracellular ATP levels. Front. Endocrinol. (Lausanne) 3:41, 2012.

${ }^{5}$ Combs, C. E., et al. Urocortin is a novel regulator of osteoclast differentiation and function through inhibition of a canonical transient receptor potential 1-like cation channel. J. Endocrinol. 212(2):187-197, 2012.

${ }^{6}$ Everaerts, W., B. Nilius, and G. Owsianik. The vanilloid transient receptor potential channel TRPV4: from structure to disease. Prog. Biophys. Mol. Biol. 103(1):2-17, 2010.
}

${ }^{7}$ Faccio, R., and V. Cremasco. PLCgamma2: where bone and immune cells find their common ground. Ann. N. Y. Acad. Sci. 1192:124-130, 2010.

${ }^{8}$ Fahlgren, A., et al. Fluid pressure and flow as a cause of bone resorption. Acta Orthop. 81(4):508-516, 2010.

${ }^{9}$ Hazama, R., et al. ATP-induced osteoclast function: the formation of sealing-zone like structure and the secretion of lytic granules via microtubule-deacetylation under the control of Syk. Genes Cells 14(7):871-884, 2009.

${ }^{10}$ Henriksen, Z., et al. The predominant mechanism of intercellular calcium wave propagation changes during long-term culture of human osteoblast-like cells. Cell Calcium 39(5):435-444, 2006.

${ }^{11}$ Hiken, J. F., and T. H. Steinberg. ATP downregulates P2X7 and inhibits osteoclast formation in RAW cells. Am. J. Physiol. Cell Physiol. 287(2):C403-C412, 2004.

${ }^{12}$ Huo, B., X. L. Lu, and X. E. Guo. Intercellular calcium wave propagation in linear and circuit-like bone cell networks. Philos. Trans. A Math. Phys. Eng. Sci. 2010(368): 617-633, 1912.

${ }^{13}$ Huo, B., et al. Fluid flow induced calcium response in bone cell network. Cell. Mol. Bioeng. 1(1):58-66, 2008.

${ }^{14}$ Huo, B., et al. An ATP-dependent mechanism mediates intercellular calcium signaling in bone cell network under single cell nanoindentation. Cell Calcium 47:234-241, 2010.

${ }^{15}$ Hwang, S. Y., and J. W. Putney. Orail-mediated calcium entry plays a critical role in osteoclast differentiation and function by regulating activation of the transcription factor NFATc1. FASEB J. 26(4):1484-1492, 2012.

${ }^{16}$ Ilvesaro, J., and J. Tuukkanen. Gap-junctional regulation of osteoclast function. Crit. Rev. Eukaryot. Gene Expr. 13(2-4):133-146, 2003.

${ }^{17}$ Johansson, L., et al. Bone resorption induced by fluid flow. J. Biomech. Eng. Trans. ASME 131(9):094505, 2009.

${ }^{18}$ Kajiya, H. Calcium signaling in osteoclast differentiation and bone resorption. Adv. Exp. Med. Biol. 740:917-932, 2012.

${ }^{19} \mathrm{Kim}, \mathrm{K}$., et al. The transmembrane adaptor protein, linker for activation of T cells (LAT), regulates RANKL-induced osteoclast differentiation. Mol. Cells 33(4):401-406, 2012.

${ }^{20}$ Kuroda, Y., et al. Osteoblasts induce $\mathrm{Ca} 2+$ oscillationindependent NFATc1 activation during osteoclastogenesis. Proc. Natl. Acad. Sci. U.S.A. 105(25):8643-8648, 2008.

${ }^{21}$ Lemaire, I., S. Falzoni, and E. Adinolfi. Purinergic signaling in giant cell formation. Front. Biosci. (Elite Ed.) $4: 41-55,2012$

${ }^{22}$ Lev, S., et al. Signal dependent hydrolysis of PI(4,5)P2 without activation of phospholipase $\mathrm{C}$ : implications on the gating of the Drosophila TRPL channel. J. Biol. Chem. 287:1436-1447, 2011.

${ }^{23} \mathrm{Li}$, P., et al. Fluid flow-induced calcium response in early or late differentiated osteoclasts. Ann. Biomed. Eng. 40(9): 1874-1883, 2012.

${ }^{24}$ Liu, B., et al. Two distinct phases of calcium signalling under flow. Cardiovasc. Res. 91(1):124-133, 2011.

${ }^{25} \mathrm{Lu}, \mathrm{X}$. L., et al. Calcium response in osteocytic networks under steady and oscillatory fluid flow. Bone 51(3):466-473, 2012.

${ }^{26} \mathrm{Lu}, \mathrm{X}$. L., et al. Osteocytic network is more responsive in calcium signaling than osteoblastic network under fluid flow. J. Bone Miner. Res. 27(3):563-574, 2012.

${ }^{27}$ Masuyama, R., et al. TRPV4-mediated calcium influx regulates terminal differentiation of osteoclasts. Cell Metab. 8(3):257-265, 2008. 
${ }^{28}$ Matemba, S. F., A. Lie, and M. Ransjo. Regulation of osteoclastogenesis by gap junction communication. J. Cell. Biochem. 99(2):528-537, 2006.

${ }^{29}$ Mentaverri, R., et al. The calcium sensing receptor is directly involved in both osteoclast differentiation and apoptosis. FASEB J. 20(14):2562-2564, 2006.

${ }^{30}$ Miyazaki, T., et al. Intracellular and extracellular ATP coordinately regulate the inverse correlation between osteoclast survival and bone resorption. J. Biol. Chem. 287(45):37808-37823, 2012.

${ }^{31}$ Mizoguchi, F., et al. Transient receptor potential vanilloid 4 deficiency suppresses unloading-induced bone loss. J. Cell. Physiol. 216(1):47-53, 2008.

${ }^{32}$ Nishii, N., et al. Effects of ATP on the intracellular calcium level in the osteoblastic TBR31-2 cell line. Biol. Pharm. Bull. 32(1):18-23, 2009.

${ }^{33}$ Noh, A. L., et al. L-type $\mathrm{Ca}(2+)$ channel agonist inhibits RANKL-induced osteoclast formation via NFATc1 downregulation. Life Sci. 89(5-6):159-164, 2011.

${ }^{34} \mathrm{O}$ 'Neil, R. G., and S. Heller. The mechanosensitive nature of TRPV channels. Pflugers Archiv. (Eur. J. Physiol.) 451(1):193-203, 2005.

${ }^{35}$ Reyes, J. P., S. M. Sims, and S. J. Dixon. P2 receptor expression, signaling and function in osteoclasts. Front. Biosci (Sch. Ed.) 3:1101-1118, 2011.

${ }^{36}$ Sakai, H., et al. Phospholipase C-dependent $\mathrm{Ca} 2+$ -sensing pathways leading to endocytosis and inhibition of the plasma membrane vacuolar $\mathrm{H}+$-ATPase in osteoclasts. Am. J. Physiol. Cell Physiol. 299(3):C570-C578, 2010 .
${ }^{37}$ Schilling, A. F., et al. Gap junctional communication in human osteoclasts in vitro and in vivo. J. Cell Mol. Med. 12(6A):2497-2504, 2008.

${ }^{38}$ Son, A., et al. Effects of inositol 1,4,5-triphosphate on osteoclast differentiation in RANKL-induced osteoclastogenesis. Korean J. Physiol. Pharmacol. 16(1):31-36, 2012.

${ }^{39}$ Sukharev, S., and D. P. Corey. Mechanosensitive channels: multiplicity of families and gating paradigms. Sci. STKE 2004(219):re4, 2004.

${ }^{40}$ Thompson, W. R., et al. Association of the alpha(2)delta(1) subunit with $\mathrm{Ca}(\mathrm{v}) 3.2$ enhances membrane expression and regulates mechanically induced ATP release in MLO-Y4 osteocytes. J. Bone Miner. Res. 26(9):2125-2139, 2011.

${ }^{41}$ Tsuzuki, T., et al. Osmotic membrane stretch increases cytosolic $\mathrm{Ca}(2+)$ and inhibits bone resorption activity in rat osteoclasts. Jpn. J. Physiol. 50(1):67-76, 2000.

${ }^{42}$ Wiebe, S. H., S. M. Sims, and S. J. Dixon. Calcium signalling via multiple $\mathrm{P} 2$ purinoceptor subtypes in rat osteoclasts. Cell. Physiol. Biochem. 9(6):323-337, 1999.

${ }^{43}$ Wiltink, A., et al. Cell membrane stretch in osteoclasts triggers a self-reinforcing $\mathrm{Ca} 2+$ entry pathway. Pflugers Archiv. (Eur. J. Physiol.) 429(5):663-671, 1995.

${ }^{44}$ Xia, S. L., and J. Ferrier. Calcium signal induced by mechanical perturbation of osteoclasts. J. Cell. Physiol. 163(3):493-501, 1995.

${ }^{45}$ Xia, S. L., and J. Ferrier. Localized calcium signaling in multinucleated osteoclasts. J. Cell. Physiol. 167(1):148-155, 1996.

${ }^{46}$ Yasuda, E., et al. Decreased expression of phospholipase C-beta 1 protein in endoplasmic reticulum stress-loaded neurons. Biol. Pharm. Bull. 31(4):719-721, 2008. 